\title{
Deformable Image Registration using Spring Mass System
}

\author{
Jian-Kun Shen, Bogdan J. Mastuszewski, Lik-Kwan Shark \\ Applied Digital Signal and Image Processing Research Centre, \\ University of Central Lancashire, Preston, UK \\ \{jkshen1 | bmastuszewski1 | 1shark\}@uclan.ac.uk North Western Medical Physics, Christie Hospital NHS Trust, Manchester, UK \\ Christopher J. Moore Chris.Moore@physics.cr.man.ac.uk
}

\begin{abstract}
The paper describes a novel multi-resolution registration method. It is fast, robust and offers high registration accuracy. The algorithm models deformations using an elastic spring mass system, which contains sparse masses interconnected by springs. The proposed method uses data intensity values to guide deformation with local constraints imposed by interaction of interconnecting springs. Moreover, by using such system prior information about the data can by easily embedded into the system to improve the registration accuracy. The performance of the method is tested using simulated as well as real dynamic magnetic resonance image $d M R I$ data.
\end{abstract}

\section{Introduction}

Image registration is one of fundamental tasks in image processing. This task can be simply considered as a process of aligning/matching two or more images having similar contents in some sense. For example, the images could have been captured at different times, from different viewpoints and/or using different types of sensors. Image registration has been researched extensively in the last twenty years. Whilst global rigid/affine registration has matured, deformable registration is still under intense research, specially in the biomedical image processing [5] [6].

The image registration methods can be broadly divided into two main categories, feature-based and intensity-based registration methods. The feature-based registration methods requires pre-processing step to extract corresponding image features such as points, lines and curves. By matching the corresponding image features, deformation of the whole image can be calculated using one of "smooth" interpolation methods. The often used interpolation methods are B-spline [7], Thin-Plate Spline [3], Radial Basis Functions [1], Inverse Distance Weighted Interpolation [15] or their combination [8]. The intensity-based methods operate directly on image intensity values. One of the most popular methods is to calculate the transformation using a set of equally spaced sparse control points, which are not linked to any specific image features, and finding the extreme in the 
cost function defined in the neighbourhood of the control points. Subsequently, the image deformations are calculated from displacement of sparse control points using one of interpolation methods mentioned above. This technique is often called as the Free Form Deformation [9]. As the number of control points might be significant additional regularisation, measures are necessary to avoid excessive variation of the deformation field. Rueckert et. al. [12] applied the global affine transformation first, and subsequently used the B-spline interpolation and a penalty function which is a $3 \mathrm{D}$ counterpart of the $2 \mathrm{D}$ bending energy of the Thin-Plate Spline. Schnabel et. al. [13] extended and generalised the work described in [12] by introducing multi-resolution optimisation and allowing non-uniform distribution of control points. Another often used method is to model the displacement field by using physical analogies. One of the first such methods was the scheme using the Navier-Lam Partial Differential Equations (PDEs) to model elastic behaviour of the registered data [2]. This method performs well when dealing with relatively small deformation, but is not suitable for large deformation. Christensen et. al. [4] proposed to use the Navier-Stokes PDEs to register the data with large displacements. This technique is so-called fluid registration. However, the technique has higher probability of the gross miss-registration than elastic registration. This kind of miss-registration issue has been recently addressed by Modersitzki [11], who proposed a new PDEs registration scheme, so-called diffusion registration. Since the intensity-based methods estimate the transformation based on the entire image contents, these methods are more computationally demanding. Whilst feature-based methods can greatly reduce computational complexity, they also have number of disadvantages including: (i) the process of selecting ground control points represented in the image is time consuming; (ii) the feature extraction methods produce inherent erroneous features due to imperfection and noise in the image acquired; (iii) the registration errors can be significantly increased by uneven feature distribution.

This paper describes a hybrid method, which combines feature-based and intensitybased methods. In the proposed method, the deformation is modelled using an elastic spring mass system, containing a number of sparse spring-masses and springs interconnecting them. At the initial stage the system of interconnected masses is assumed to be in equilibrium. One of the advantages of using such a system is that the system can explicitly represent features presented in the image in order to improve the registration accuracy. For instance, spring-masses can coincide with all the feature points; and also, feature curves can be represented as spring-masses placed on the curve and their interconnecting springs lying along the curve with controllable approximation error. By moving these masses representing the features in one image to the corresponding location in the other image, the system will no longer be in equilibrium. The remaining masses will move to the appropriate location until all the internal forces are in equilibrium. The motion of the spring mass system is described here by the Newtonian mechanics and is expressed by a set of ordinary differential equations, which is comparatively much simpler to solve than PDEs. In the following stage, the external forces calculated from the image content are introduced into the system to locally guide movable masses to gradually improve registration accuracy under the constraints caused by the internal forces. The applied multi-resolution approach reduces the computational time and improves the registration accuracy.

The remainder of the paper is organised as follows: Section 2, 3 and 4 briefly describe three main steps in the algorithm. They are spring mass system generation, motion modelling, and image warping. Section 5 describes a multi-resolution registration scheme. Section 6 gives some registration results. The conclusion is drawn in the section 7 . 


\section{Spring Mass System Generation}

The spatial topology of the spring mass system is defined using a mesh generation algorithm with the mesh vertices interpreted as the spring-masses and the mesh edges as the springs connecting these masses. To achieve high registration accuracy, the mesh generation algorithm should provide:

- Well shaped triangles (all triangles should have three inner-angles close to $60^{\circ}$ ) to reduce sensitivity to the mesh orientation;

- Variable mesh density that is controllable by a user;

- Vertices coinciding with all feature landmarks;

- Good representation of the feature curve (such as anatomical structures) with the vertices placed on the curve and mesh edges lying along the curve with controllable approximation errors.

Any mesh generation algorithms can be used as long as they could provide these requirements. In this paper, the bubble mesh algorithm [16] has been used to generate the mesh vertices. This method is based on the geometrical circle packing, with the centre of each circles (bubble) representing a mesh vertex. The method adapts automatically a local population of the bubbles to ensure the required local density of the mesh; and thereby, it enables generation of the well shaped triangles. The Quad-tree technique is used to achieve fast initialisation of the location of bubble centres in the image, as well as a constraint surface being used to control the sizes of the bubbles locally. Once the algorithm terminates the bubble centres (mesh vertices) are connected using Delaunay Triangulation, which selects the best topological connection for a given set of vertices by minimising the number of badly shaped triangles. With Constrained Delaunay Triangulation technique, it enforces the consistency of the mesh along the feature curves.

\section{Motion Modelling}

The motion of the system is described here by the Newtonian mechanics and is expressed by a set of the 2-nd order Ordinary Differential Equations (ODEs). The 4-th order RungeKutta method has been used to yield the numerical solution.

$$
m \frac{d^{2} \mathbf{p}_{(i)}}{d t^{2}}+c \frac{d \mathbf{p}_{(i)}}{d t}=a(t) \cdot \mathbf{f}_{i n t(i)}+b \cdot \mathbf{f}_{\text {ext }(i)} \quad \wedge_{i \in \mathscr{N}}^{\wedge}
$$

where:

- $\mathscr{N}$ is a set of indices for which the corresponding masses in the spring mass system are movable.

- $\mathbf{p}_{(i)}=\left[x_{i}, y_{i}\right]^{T}$ is the position of the $i$-th movable spring-mass.

- $m \frac{d^{2} \mathbf{p}_{(i)}}{d t^{2}}$ is the inertial force for the $i$-th movable spring-mass. The constant $m$ is the mass of the spring-mass. It is assumed that all the spring-masses have the same mass $(m=1)$. 
- $c \frac{d \mathbf{p}_{(i)}}{d t}$ is the damping force of the movable mass. The constant $c$ is damping coefficient. It is assumed that all the spring-masses has the same damping coefficient $(c=2)$.

- $\mathbf{f}_{\text {int }(i)}$ is the internal force caused by the springs connected to the $i$-th spring-mass.

- $\mathbf{f}_{\text {ext }(i)}$ is the external force acting on the $i$-th spring-mass.

- $a(t)$ is iteration dependant scalar which weights the contribution of the internal and external forces. Initially the value of scalar $a(t)$ is set to one. Subsequently, after the external force is activated, the value of this parameter is decreased gradually, after each iteration, from one to a predefined threshold $a_{0}$ (see Algorithm 1).

- $b$ is a binary value which controls whether the external forces are acting on the movable spring-masses. In the initial stage, $a(t)$ is set to one and $b$ is set to zero to disable the external forces. Subsequently, $b$ is set to one to enable the forces and $a(t)$ starts to decrease. Consequently, the external forces can guide the system deformation under the constraints caused by the internal forces. Initially, the deformation is more likely to be rigid. With the decreasing internal forces, the system gradually allows the more flexible deformation.

\subsection{Internal Forces}

The role of internal forces in the system is to make sure the calculated deformation is "smooth". In the algorithm, the repulsive force in the traditional spring force formula [10] can cause unexpected results when the deformable images contain large local movement. Therefore, the repulsive force needs to be removed. To keep the system stable in the initial stage, the extra attractive force is added locally along each spring direction. If a set of indices for which the corresponding masses are connected to the current $i$-th mass is defined as $\mathscr{M}(i)$, the internal force [14] can be written in general as:

$$
\mathbf{f}_{\text {int }(i)}=\sum_{j \in \mathscr{M}(i)} k_{j} \cdot \frac{\mathbf{p}_{j}-\mathbf{p}_{i}}{\left\|\mathbf{p}_{j}-\mathbf{p}_{i}\right\|} \cdot\left(\left\|\mathbf{p}_{j}-\mathbf{p}_{i}\right\|+\rho_{j i}\right) \quad \wedge_{j \in \mathscr{M}}^{\wedge}
$$

where:

- $k_{j}$ is a spring constant locally controlling how easily the system can be deformed. The default value is one.

- $\rho_{j i}$ is the "added" length for the current $i$-th mass along the spring direction. It is important to notice that $\mathrm{n}$ general $\rho_{j i} \neq \rho_{i j}$.

The scalars $\rho_{j i}$, where $j \in \mathscr{M}(i)$, need to be obtained. However, there are infinite possible selections of $\rho_{j i}$, which fulfil the equation in (2). Hence, a constraint is added in order to have a unique result. The constraints optimisation used here is defined by the following cost function:

$$
\underset{\rho_{j i}}{\arg \min }\left(\sum_{j \in \mathscr{M}(i)}\left(k_{j} \cdot \rho_{j i}\right)^{2}\right)
$$


subject to two equality constraints in (4) and $N$ inequality constraints in (5)

$$
\begin{gathered}
\mathbf{f}_{\text {int }(i)}=0 \\
\rho_{j i} \geq 0, \quad \text { where } j=1 \ldots N
\end{gathered}
$$

This can be solved using Lagrange Multiplier method [14].

\subsection{External Forces}

The external force defined as $\mathbf{f}_{\text {ext(i) }}$ is a force indicating how much the $i$-th spring-mass should be moved towards the good matching direction. The role of external forces is to incorporate information of image content into the system to achieve iteratively higher and higher registration accuracy. In this case, a small size image $I_{i}^{w}$ is extracted from the warped source image, whose centre is the current location of the $i$-th spring-mass, to estimate the good local matching direction in the target image $I_{i}^{t}$ around the corresponding location of the $i$-th spring-mass. The external force is defined as:

$$
\mathbf{f}_{\text {ext }(i)}=\beta \cdot \operatorname{grad}\left(f\left(d\left(I_{i}^{w}, I_{i}^{t}\right)\right)\right)
$$

where:

- $d(\cdot, \cdot)$ is a similarity measure between two images.

- $I_{i}^{w}$ and $I_{i}^{t}$ are the $i$-th windows taken out respectively from the warped source and target images.

- $\beta$ is a measure of the reliability of $\operatorname{grad}\left(f\left(d\left(I_{i}^{w}, I_{i}^{t}\right)\right)\right)$.

The proposed algorithm enables the use of different similarity measures. For the results presented in this paper, the normalised cross correlation function in (7) with the experimentally selected parameters $(A=50$ and $n=2)$ has been used. However, it can be replaced by other measures such as sum of square differences or mutual information.

$$
f(d(\cdot, \cdot))=\left\{\begin{array}{cc}
A \cdot(d(\cdot, \cdot))^{n} & d(\cdot, \cdot) \geq 0 \\
0 & d(\cdot, \cdot)<0
\end{array}\right.
$$

\section{Image Warping}

After the solution of equations defined by formula (1) has been found, the source image needs to be warped to match the target image. This process involves coordinate transformation to find corresponding locations of all the pixels from the warped source image in the source image (inverse mapping is used) and subsequent computation of the pixel values in these new locations. while the standard bilinear interpolation is used for the latter, one of interpolation methods for scattered data needs to be used for the former. In the proposed method, the image warping step needs to be used iteratively. Therefore, the piecewise affine interpolation based on Constrained Delaunay Triangulation [14] is used.

Figure 1 illustrates an example of motion modelling and image warping using the spring mass system with three rigid structures. The boundaries of the image and three structures are represented as the point masses denoted by the asterisks. At this stage, 


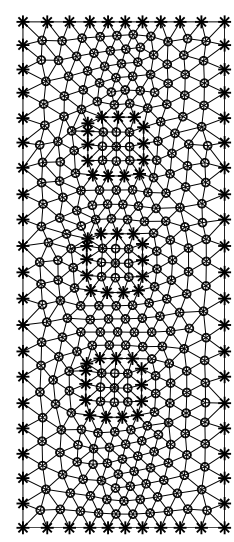

(a)

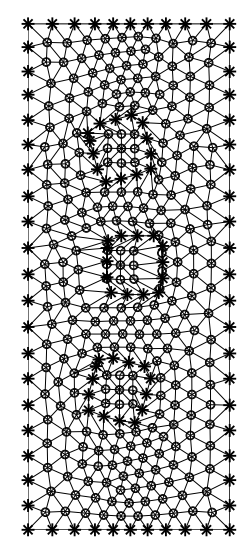

(b)

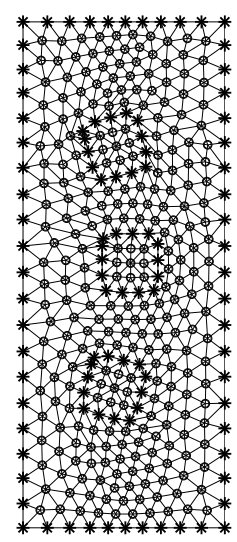

(c)

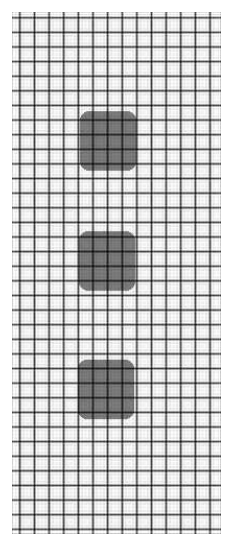

(d)

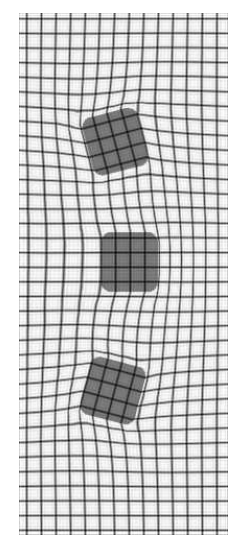

(e)

Figure 1: Example of spring mass system deformation

the system as shown in Figure 1(a) is in equilibrium. By moving masses representing the three structures to the new location as shown in Figure 1(b), the system is no longer stable. Without the external forces applied, the movable masses denoted as circles move to the appropriate location until all the internal forces are in equilibrium. The solution is shown in Figure 1(c). To have a better illustration for transformation in the whole image domain, Figure 1(e) shows a warped texture using the source texture in the Figure 1(d).

\section{Multi-resolution Registration Scheme}

The multi-resolution approach [2] [13] is an often used technique to register the images with larger deformation. In the work presented in the paper, a Gaussian pyramid is built for the source and target images first. Subsequently, the registration scheme starts at the coarsest level and progress to the finest level. The multi-resolution registration scheme is summarised in Algorithm 1. In the work, $d \_$ratio is set to 0.8 and the threshold $a_{0}$ is equal to 0.3 .

\section{Results and Validation}

The proposed method is first tested using simulated data. The 512-by-512 source image shown in Figure 2 is warped using a sequence of randomised transformations to generate a set of simulated target images (see Figure 2). Subsequently, the described registration algorithm is used to recover the known displacement field. To generate these simulated target images, the bony structures and the rectum have been segmented in the source image and each of them have been transformed rigidly with randomly selected translation vector (with maximum translation of \pm 20 pixels) and rotation angle (with maximum of $\pm 15^{\circ}$ ). The simulated images are generated using Little's Radial Basis Function method 


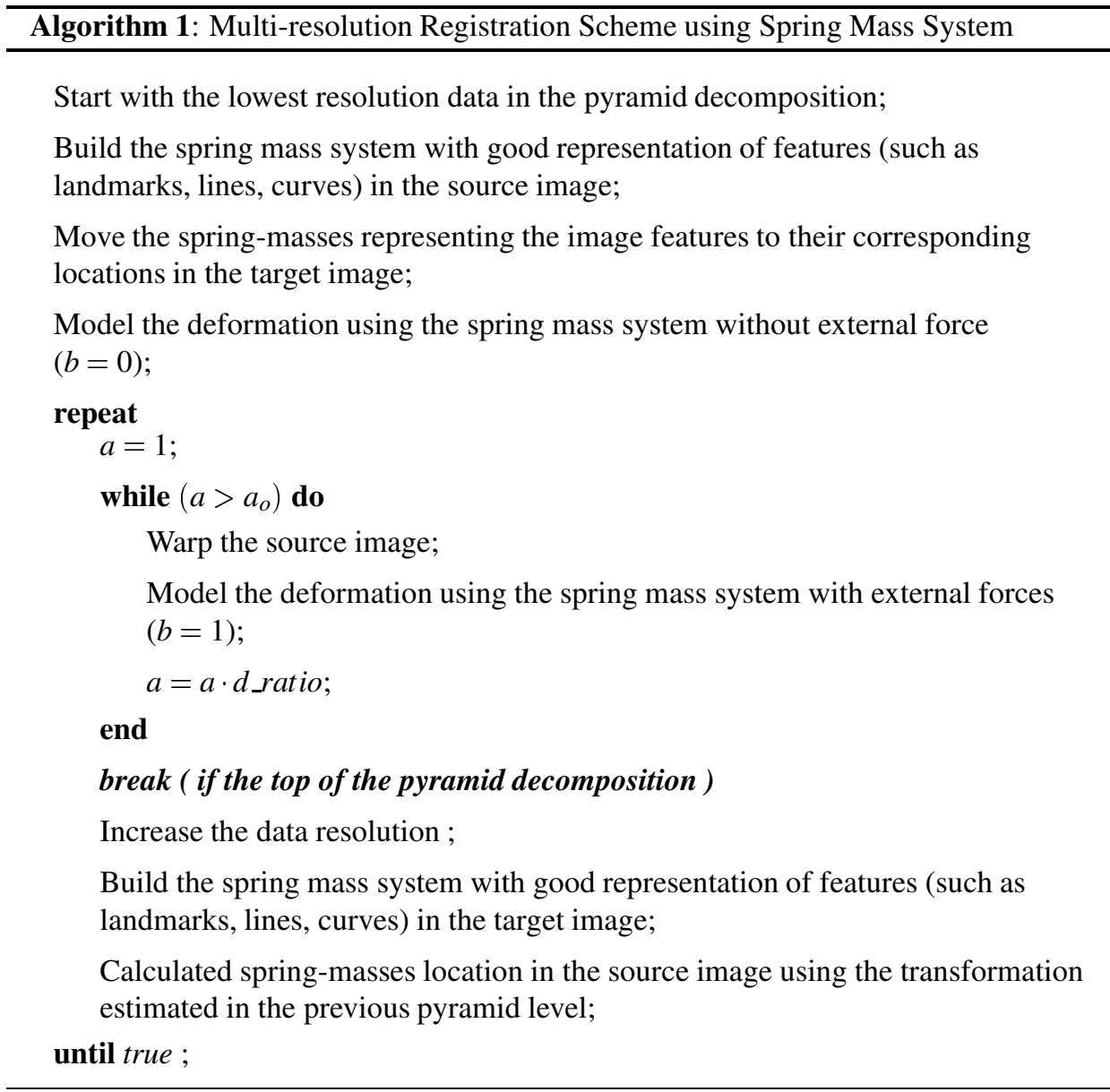

\section{described in [8].}

Figure 3 shows typical results obtained using the multi-resolution registration technique described in the previous section. A simple statistics of the displacement field calculated for each resolution level are shown in Table 1. The column with bold letters in Table 1 represents statistics calculated for the original simulated displacement field. The results shown in Figure 3 and Table 1 are typical for the all the simulated target images as described at the beginning of this section.

The proposed method has been also applied for a sequence of real dynamic magnetic resonance images. The source and target images are randomly selected from the sequence. Four of such examples are shown in Figure 4.

\section{Conclusion}

The paper presents a new registration method using an elastic spring mass system, which enables to reflect prior information about image including spatial topology of image fea- 

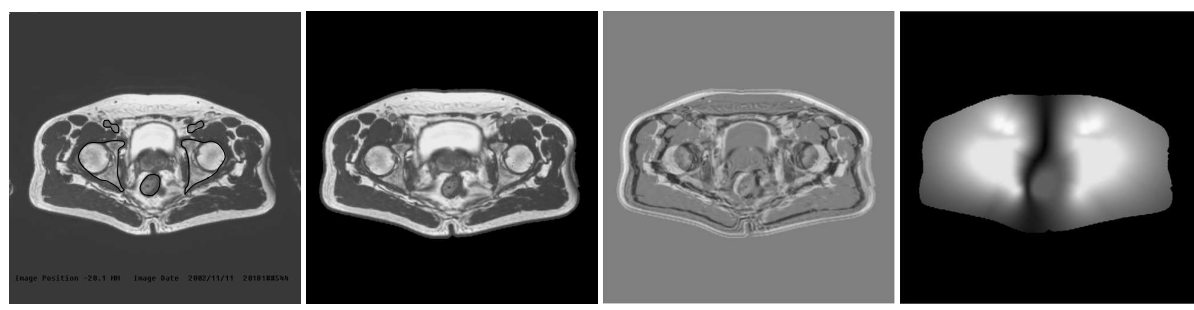

Figure 2: From left to right: source image with five segmented structures used for the simulation of the target image; one of simulated target images; difference between source and simulated target images; displacement field between source and simulated target images.
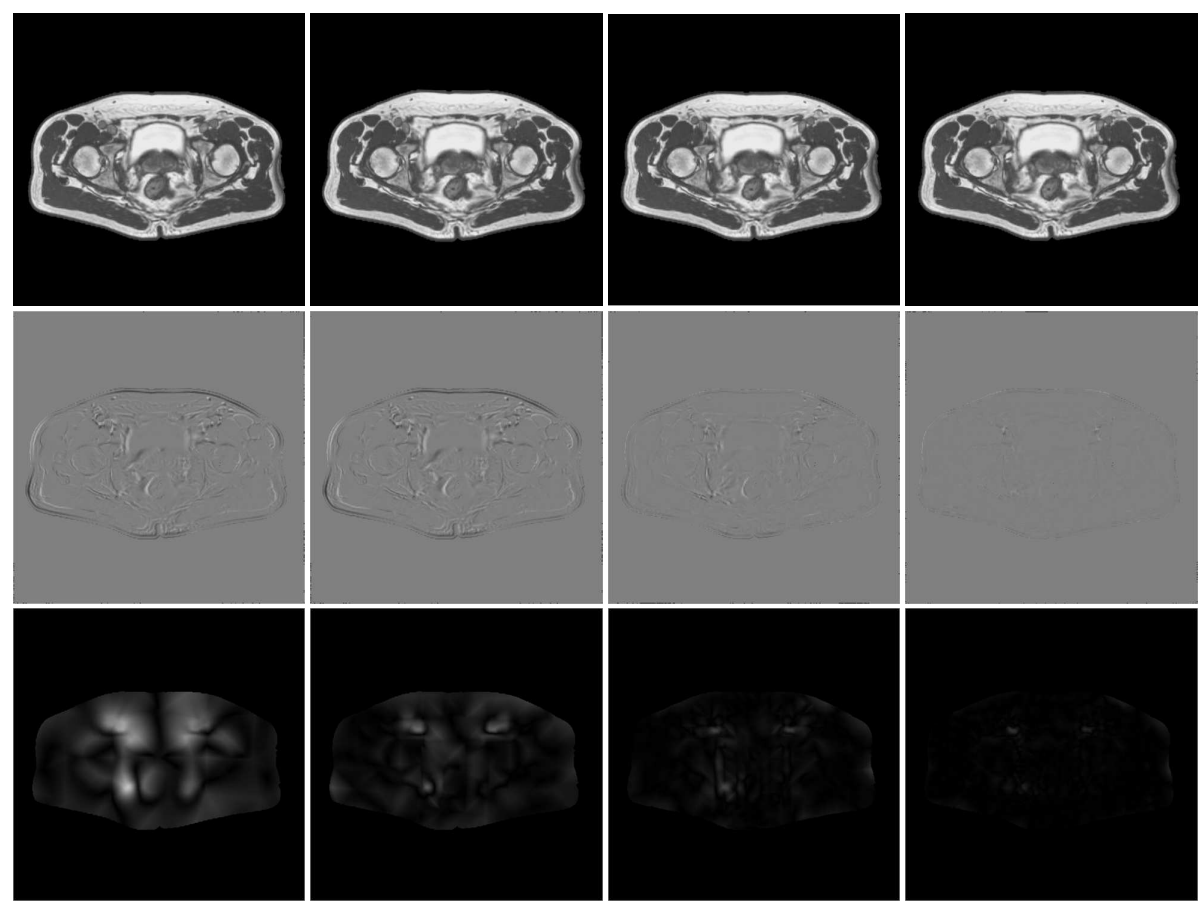

Figure 3: From top to bottom: registered source image, difference between registered and simulated target images, displacement field between registered and simulated target images. The results have been obtained at different resolution levels, as described in section 5 with the columns from left to right showing results obtained with $1 / 8,1 / 4,1 / 2$ and full resolution levels.

\begin{tabular}{cccccc} 
& Before & $1 / 8$ resolution & $1 / 4$ resolution & $1 / 2$ resolution & full resolution \\
\hline max & $\mathbf{2 2 . 2 6 0 7}$ & 15.2603 & 8.1379 & 5.3598 & 3.9656 \\
mean & $\mathbf{1 2 . 8 5 4 0}$ & 2.8896 & 1.4601 & 0.6671 & 0.2832 \\
std & $\mathbf{5 . 3 5 0 9}$ & 2.2378 & 1.0104 & 0.5703 & 0.3006 \\
\hline
\end{tabular}

Table 1: Validation results using simulated images 

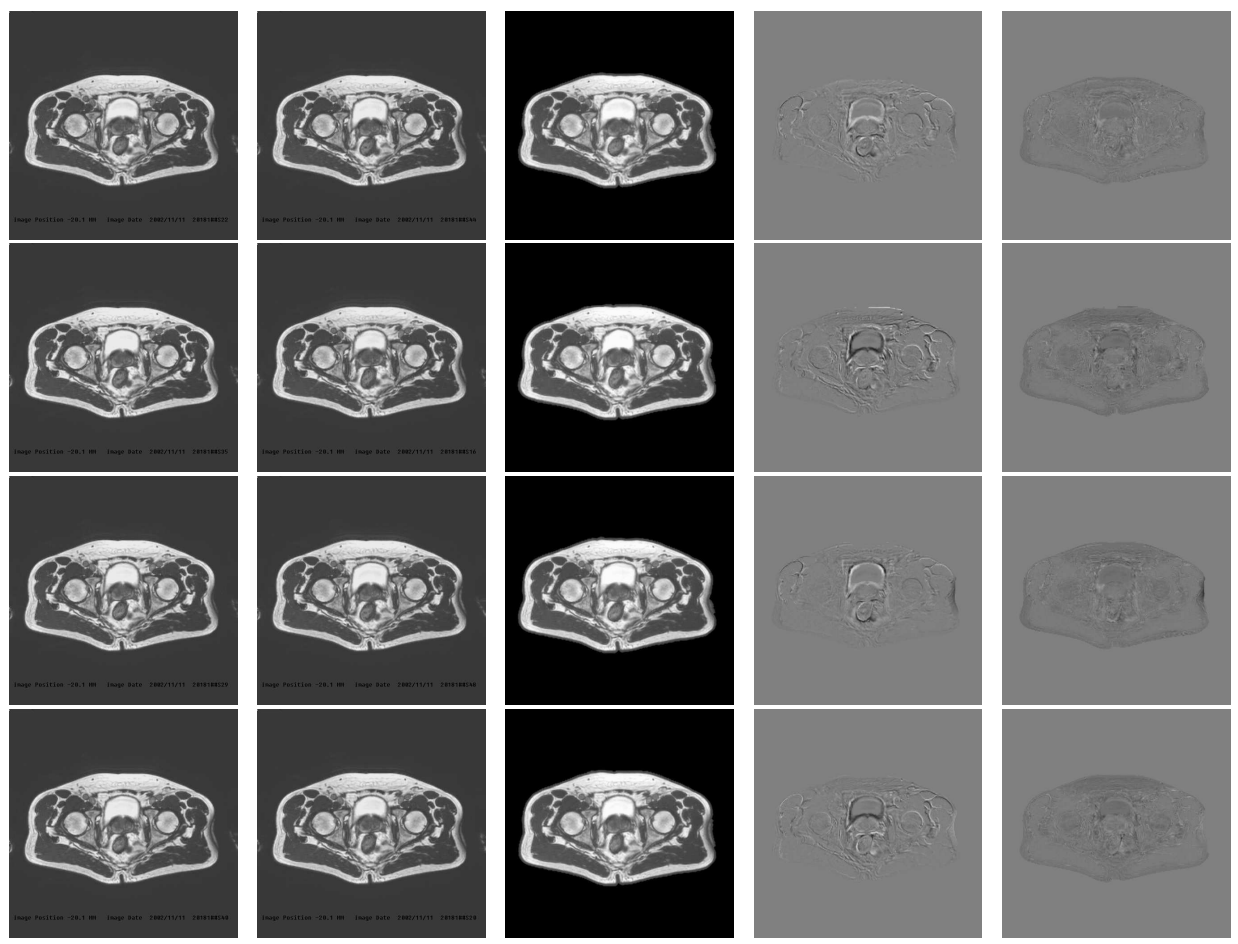

Figure 4: The columns from left to right show: the source images; the target images; the registered target images; image differences between the source and target images; image differences between the registered and target images.

tures and local elastic properties. By moving the masses representing the features in one of the images to the corresponding location in the other image, an initial deformation is modelled via the internal forces caused by springs. Subsequently, the external forces are calculated from image content and embedded into the system. This guides the system to achieve higher and higher registration accuracy under the constraints of the internal forces. With the multi-resolution registration scheme, it improves registration quality for the large deformation. Although the registration results have been shown only using $d M R I$ data in the same modality, the algorithm can generally be used to register multi-modality and multi-dimension data.

\section{References}

[1] N. Arad and D. Reisfeld. Image warping using few anchor points and radial functions. Computer Graphics Forum, 14:35-46, 1995.

[2] R. Bajcsy and S. Kovacic. Multiresolution elastic matching. Computer Vision, Graphics and Image Processing, 46:1-21, 1989. 
[3] F.L. Brookstein. Principal warps: Thin-plate splines and the decomposition of deformations. IEEE Transaction on Pattern Analysis and Machine Intelligence, 11:567$585,1989$.

[4] G.E. Christensen, R.D. Rabbitt, and M.I. Miller. Deformable templates using large deformation kinematics. IEEE transactions on Image Processing, 5:1434-1447, 1996.

[5] W.R. Crum, T. Hartkens, and D.L.G. Hill. Non-rigid image registration: Theory and practice. The British Journal of Radiology, 77:140-143, 2004.

[6] D.J. Hawkes, D. Barratt, J.M. Blackall, C. Chan, P.J. Edwards, K. Rhode, G.P. Penney, J. McClelland, and D.L. Hill. Tissue deformation and shape models in image-guided interventions: A discussion paper. Medical Image Analysis, 9:163$175,2005$.

[7] S. Lee, G. Wolberg, and S.Y. Shin. Scattered data interpolation with multilevel bsplines. IEEE Transactions on Visualization and Computer Graphics, 3:228-244, 1997.

[8] J.A. Little, D.L.G. Hill, and D.J. Hawkes. Deformations incorporating rigid structures. Computer Vision and Image Understanding, 66:223-232, 1997.

[9] R. MacCraken and K. Joy. Free form deformations with lattices of arbitrary topology. Proceedings of ACM SIGGRAPH, pages 181-188, 1996.

[10] B.J. Matuszewski, J.-K. Shen, and L.-K. Shark. Elastic image matching with embedded rigid structures using spring mass system. IEEE International Conference on Image Processing, 10:937-940, 2003.

[11] J. Modersitzki. Numerical Methods for Image Registration. Oxford University Press, New York, 2004.

[12] D. Rueckert, L.I. Sonoda, C. Hayes, D.L. Hill, M.O. Leach, and D.J. Hawkes. Nonrigid registration using free form deformations: Application to breast mr images. IEEE Transactions on Medical Imaging, 18:712-721, 1999.

[13] J.A. Schnabel, D. Rueckert, M. Quist, J.M. Blackall, A.D. Castellano Smith, T. Hartkens, G.P. Penney, W.A. Hall, H. Liu, C.L. Truwit, F.A. Gerritsen, D.L.G. Hill, and D.J. Hawkes. A generic framework for nonrigid registration based on nonuniform multi-level free form deformations. Conf. on Medical Image Computing and Computer-Assisted Intervention, pages 573-581, 2001.

[14] J.-K. Shen, B. J. Matuszewski, and L.-K. Shark. Deformable image registration. IEEE International Conference on Image Processing, 3:1112-1115, 2005.

[15] D. Shepard. A two dimensional interpolation function for irregularly spaced data. ACM in Proceeding 23 National Conference, pages 517-524, 1968.

[16] K. Shimada and D.C. Gossard. Bubble mesh: Automated triangular meshing of nonmanifold geometry by sphere packing. ACM 3rd Symposium on Solid Modelling and Applications, pages 409-419, 1995. 